\title{
Gain-of-Function Mutation Met136Val in SCN8A is not a Common Cause of Trigeminal Neuralgia
}

\author{
Raymond Sekula $^{1}$, Kathleen Deeley ${ }^{1}$, Hayley Denwood ${ }^{1}$, and Alexandre Vieira ${ }^{1}$ \\ ${ }^{1}$ University of Pittsburgh
}

May 5, 2020

\begin{abstract}
Classical trigeminal neuralgia (cTN) is a neuropathic pain disorder marked by evoked and spontaneous attacks in the distribution of the trigeminal nerve, and the disorder is further associated with periods of complete remission and subsequent recurrence in most patients. A mutation in SCN8A, a sodium channel gene that codes for the Nav1.6 protein, was reported in a 64 -yearold white female that presented with classical trigeminal neuralgia. The Met136Val change produced a significant increase in peak transient and resurgent currents of Nav1.6, reduced the threshold for action potential in trigeminal ganglia neurons, and enhanced the neuronal evoked response and the fraction of neurons that fire at a higher rate than those expressing wild type channels. We sequenced 123 individuals with a diagnosis of trigeminal neuralgia and did not detect the mutation, suggesting its frequency is lower than $1 \%$ in the population.
\end{abstract}

Classical trigeminal neuralgia (cTN) is a neuropathic pain disorder marked by evoked and spontaneous attacks in the distribution of the trigeminal nerve, and the disorder is further associated with periods of complete remission and subsequent recurrence in most patients. The pain of cTN is considered to be amongst the most debilitating types of pain. Neurovascular compression (NVC) of the trigeminal nerve has been accepted as the cause of cTN in a majority of patients by the International Headache Society (IHS). The condition is usually sporadic, although a recent publication suggests that familial occurrence may be more common than previously considered (2). A mutation in $S C N 8 A$, a sodium channel gene that codes for the $\mathrm{Na}_{\mathrm{v}} 1.6$ protein, was reported in a 64 -year-old white female that presented with classical trigeminal neuralgia. The Met136Val change produced a significant increase in peak transient and resurgent currents of $\mathrm{Na}_{\mathrm{v}} 1.6$, reduced the threshold for action potential in trigeminal ganglia neurons, and enhanced the neuronal evoked response and the fraction of neurons that fire at a higher rate than those expressing wild type channels (3). Mutations in $S C N 8 A$ are known to cause $S C N 8 A$-related epilepsy with encephalopathy, a rare condition that has been reported in 50 individuals worldwide, which is characterized by developmental delay, seizure onset in the first 18 months of life (mean 4 months), and intractable epilepsy characterized by multiple seizure types (4). Since it was not clear from the original report how frequent the Met136Val variant in $S C N 8 A$ is among individuals with trigeminal neuralgia, we tested the hypothesis that the mutation SCN8A Met136Val is an infrequent finding in individuals with trigeminal neuralgia.

One hundred and twenty-three individuals diagnosed with trigeminal neuralgia were recruited since January 2016 to be part of our Orofacial Pain Registry and Sample Repository project (IRB approval \# 15110027). They were 81 females, 110 Whites, and all adults at least 45 years of age. These individuals provided written informed consent and a biological sample (unstimulated saliva) and are from the most part from the western Pennsylvania region. All cases were diagnosed by the same professional (R.F.S.) using the same criteria. DNA was extracted from saliva according to a published protocol (5) and samples were sequenced in both directions to determine the presence of the SCN8AMet136Val mutation in the exon 4 of the gene. 
We designed a set of primers (5' TGT GCT TCA TCT CCT TTC AGG 3' forward and 5' CCA CAT TCT TCG ACC AGT CA 3' reverse) using the Primer3 online software (http://primer3.ut.ee/). Polymerase chain reactions (PCR) conditions were 30 cycles at $95^{\circ} \mathrm{C}$ for 30 seconds, $55^{\circ} \mathrm{C}$ for 30 seconds, and $72^{\circ} \mathrm{C}$ for 1 minute, followed by a 7 minute-hold at $72^{\circ} \mathrm{C}$. Sequences were analyzed using the DNA Analysis Software Sequencher 5.0 (Sequencher 5.0: Gene Codes, Ann Harbor, Michigan, USA; http://genecodes.com/).

We did not find any case with the SCN8A Met136Val, which suggests that the frequency of this mutation explains less than $1 \%$ of cases of trigeminal neuralgia in populations.

These findings should help guide future study designs that aim to identify associations between sodium channel genes and trigeminal neuralgia. Analyses of common variants of $\mathrm{Na}_{\mathrm{v}} 1.7(S C N 9 \mathrm{~A}$ rs6746030) and nerve growth factor receptor (SCN9Ars6746030) of 48 individuals did not show overrepresentation of alleles in cases with trigeminal neuralgia (6) and it is likely that several hundred to thousands of individuals are necessary for enough statistical power to detect associations.

Acknowledgements

The authors have no conflict of interest to declare. We thank the enthusiastic participation of all subjects.

References

1. Cruccu, G., Bonamico, L.H., \& Zakrzewska, J.M. (2010). Cranial neuralgias. The Handbook of Clinical Neurology, 97, 663-678.doi :10.1016/S0072-9752(10)97056-5

2. Di Stefano, G., Yuan, J-H., Cruccu, G., Waxman, S.G., Dib-Hajj, S.D., \& Truini, A. (2020). Familial trigeminal neuralgia - a systematic clinical study with a genomic screen of the neuronal electrogenisome.Cephalalgia [Epub ahead of print] doi :10.1177/0333102419897623

3. Tanaka, B.S., Zhao, P., Dib-Hajj, F.B., Morisset, V., Tate, S., Waxman, S.G., \& Dib-Hajj, S.D. (2016). A gain-of-function mutation in Nav1.6 in a case of trigeminal neuralgia. Molecular Medicine, 2,338-348.doi $: 10.2119 /$ molmed.2016.00131

4. Hammer, M.F., Wagnon, J.L., Mefford, H.C., Meisler, M.H., Adam, M.P., Ardinger, H.H., Pagon, R.A., Wallace, S.E., Bean, L.J.H., Stephens, K., \& Amemiya, A. (2016). SCN8A-related epilepsy with encephalopathy. GeneReviews@ [Internet]. Seattle (WA): University of Washington, Seattle, 1993-2020.

5. Deeley, K., Noel, J., \& Vieira, A.R. (2016). Comparative study of five commercially available saliva collection kits for DNA extraction. Clinical Laboratory, 62, 1809-1813.doi :10.7754/Clin.Lab.2016.160207

6. Costa, G.M.F., Rocha, L.P.C., Siqueira, S.R.D.T., Moreira, P.R., \& Almeida-Leite, C.M. (2019). No association of polymorphisms in Nav1.7 or nerve growth factor receptor genes with trigeminal neuralgia. Pain Medicine, 20, 1362-1369.doi :10.1093/pm/pny191 